\title{
Air Quality Monitoring Using Assistive Robots for Ambient Assisted Living and Enhanced Living Environments through Internet of Things
}

\author{
Gonçalo Marques ${ }^{1,2, *(\mathbb{D}}$, Ivan Miguel Pires ${ }^{2,3}$, Nuno Miranda ${ }^{1}(\mathbb{D})$ and Rui Pitarma ${ }^{1}(\mathbb{D}$ \\ 1 Research Unit for Inland Development, Polytechnic Institute of Guarda, 6300-559 Guarda, Portugal; \\ nuno-miranda@sapo.pt (N.M.); rpitarma@ipg.pt (R.P.) \\ 2 Instituto de Telecomunicações, Universidade da Beira Interior, 6201-001 Covilhã, Portugal; impires@it.ubi.pt \\ 3 Computer Science Department, Polytechnic Institute of Viseu, 3504-510 Viseu, Portugal \\ * Correspondence: goncalosantosmarques@gmail.com; Tel.: +351-926-5256-717
}

Received: 27 October 2019; Accepted: 18 November 2019; Published: 20 November 2019

\begin{abstract}
This paper presents iAirBot, an assistive robot for indoor air quality monitoring based on Internet of Things. The system can communicate with occupants and triggers alerts automatically using social networks. The information can be accessed by the caregiver to plan interventions for enhanced living environments in a timely manner. The results are promising, as the proposed architecture presents a cost-effective assistive robot for indoor quality monitoring. It connects several technological fields and knowledge areas, such as ambient assisted living, Internet of Things, wireless sensor networks, social networks, and indoor air quality. When compared to other systems, iAirBot stands out for the modularity and scalability of its sensors network, as well as the use of social networks for information sharing. Therefore, iAirBot is a significant system for enhanced living environments, occupational health, and well-being.
\end{abstract}

Keywords: indoor air quality; ambient assisted living; enhanced living environments; internet of things; smart home; social networks; wireless sensor networks

\section{Introduction}

By 2050, 20\% of the world's population will be over 60 years old, which will result in increased disease, health costs, caregiver shortages, and a significant social impact [1]. In particular, ageing in Portugal is a current phenomenon. Portugal is currently one of the five most aged countries in the world [2]. Forty years ago, it was the country with the highest birth rate in Europe, but currently it has the lowest birth rate [3,4]. Therefore, the development of assistive robots is increasingly needed worldwide, and in Portuguese society in particular. According to PORDATA, the elderly dependency ratio has increased in all European Union countries. In 2017, Portugal ranked fourth in this list, while Italy was at the top and Luxembourg was at the end [5]. Figure 1 shows the dependency ratio in Europe. The elderly dependency ratio is the number of people aged 65 and over compared to 100 working-age people aged between 15 and 64 . Consequently, the increase of dependent elderly people associated with a lower birth rate results in a significant need for the development of solutions related to enhanced living environments. 


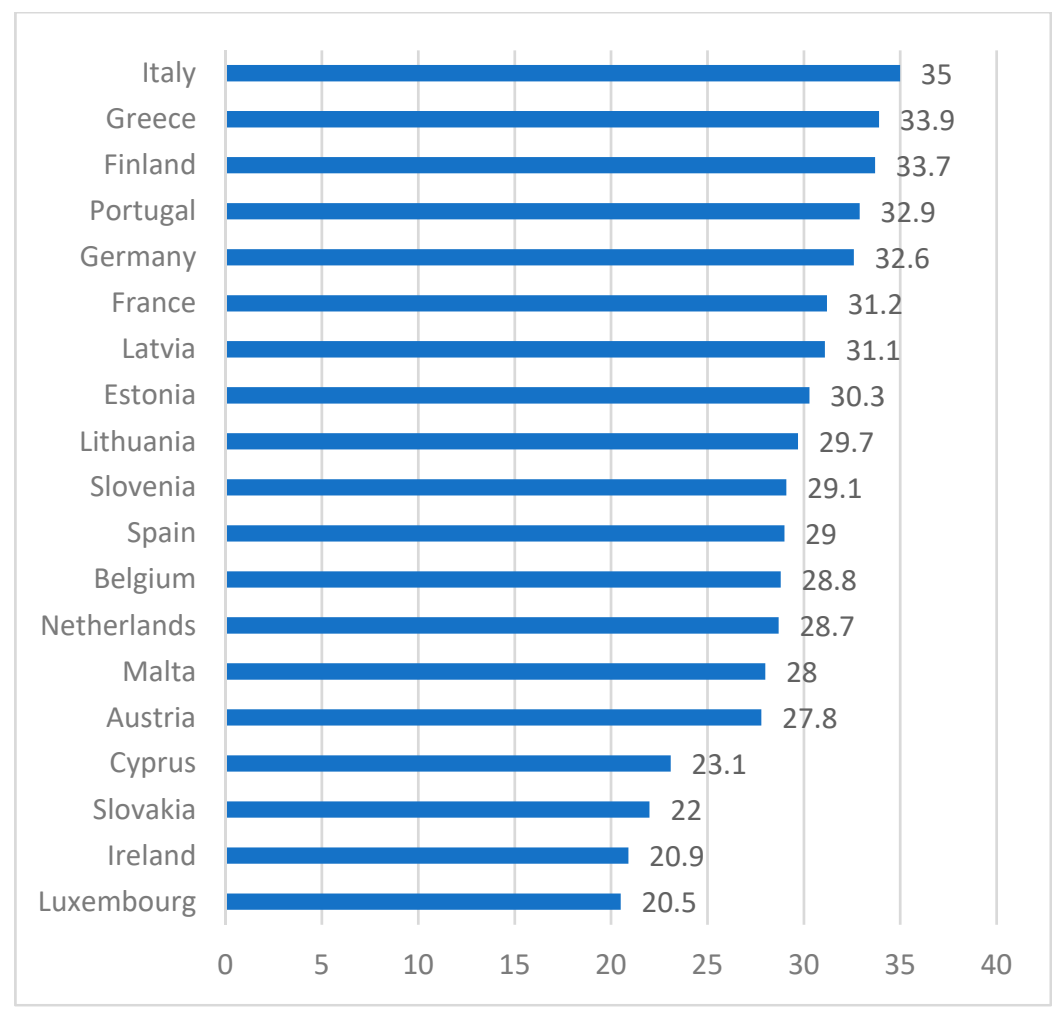

Figure 1. Index of the dependence of the elderly (2017).

Ambient assisted living (AAL) is a multidisciplinary scientific field involving the creation of high-level software and hardware systems to increase occupational health, quality of life, and well-being for enhanced living environments [6,7]. Enhanced living environments in the AAL field are closely related to information and communications technologies. An enhanced living environment incorporates enhanced systems, platforms, methods, and architectures which, directly or indirectly help people to remain living in their homes instead of been transferred to nurseries and homes for the elderly. These systems incorporate sensors, actuators, microcontrollers, wireless communication technologies, and open-source software to provide numerous healthcare services for enhanced occupational health in an effective and often pervasive manner [8]. Nevertheless, several issues still exist in the development of AAL systems for enhanced occupational health [9]. Furthermore, social and moral challenges still exist, for example, the acceptance of these technologies by older persons and privacy and security problems. Therefore, it is crucial to ensure that innovation does not replace human care, but rather presents new approaches for medical support. In total, $87 \%$ of individuals choose to remain in their homes and bear the cost of nursing instead of going into retirement homes [10]. Regarding these arguments, there is a significant need to develop automatic systems to increase the quality of life for enhanced living environments and occupational health.

In general, people spend around $90 \%$ of their time indoors. The elderly population and newborns are the most vulnerable to poor indoor air quality (IAQ), because they may spend all their time inside buildings [11]. Thus, poor IAQ is the main risk factor to which an individual is exposed [12]. In particular, poor IAQ is a challenge that affects the poorest and most vulnerable individuals in the world. It is a global health problem comparable to problems such as smoking and sexually transmitted diseases [13]. The Environmental Protection Agency (EPA) that regulates air quality in the United States recognizes that the level of indoor pollutants can be up to 100 times higher than outdoor air pollutants. Air quality is in the top five most serious environmental problems for global health [14]. There is a significant need for IAQ research to improve legislation, inspection, and to create automatic real-time monitoring solutions for enhanced living environments. Those systems should be incorporated not only in public places, such as institutions and hospitals, but also in private places to increase the 
building's construction guidelines. Simple behavioral changes such as avoidance of smoking indoors and the use of natural ventilation are important and can provide numerous positive impacts in terms of IAQ, and should be taught to children through educational programs [15]. However, in spite of IAQ effects on public health, there is a lack of interest in the research community in studying new methods for enhanced IAQ [16].

The Internet of Things (IoT) includes not only communication devices but also every other physical object on the planet that can be connected and controlled through the Internet [17]. IoT can provide ubiquitous capabilities to several applications by providing physical interaction via embedded systems to create a globally distributed network of objects that can be managed by a human being in real-time [18]. The emergence of cost-effective sensors, communication protocols, microcontrollers, actuators, and miniaturized modules provides the requirements to develop IoT architectures for ambient assisted living and enhanced living environments [19]. These cyber-physical systems support built-in communication technologies and can be connected to the Internet. Consequently, these cyber-physical systems can interact with software middleware services to address day-to-day life applications. IoT offers pervasive connectivity to automatic context-aware cyber-physical systems that act intelligently without human interaction [20]. A study by IBM (International Business Machines) stated that the development of IoT offers a foundational base for a smarter planet [21]. IoT and AAL are two closely related research areas that complement each other and have grown in tandem to solve daily human routines to promote occupational health and well-being.

Social networks have changed the nature of communication among people and have become the principal sources for connecting people based on their interests. Moreover, social network usage levels continue to increase, and the quantity of time spent on social media usage and the quantity of social content are also growing [22]. A study conducted by [23] showed positive relationships between Facebook, emotional experiences, and social resources. On the one hand, some studies present positive correlations between Facebook and life satisfaction, and show no evidence for depressive consequences. On the other hand, other studies present negative emotional outcomes, with people who spend more time on Facebook more likely to become depressed because of upward social comparison.

This paper presents an assistive robot for an IAQ monitoring solution based on IoT architecture for enhanced living environments, called iAirBot. It uses TurtleBot 2 (robot platform), wireless sensors networks (WSN), and has social network support. This system is an automated solution that allows the sharing and storage of IAQ data history throughout the Facebook social network. The iAirBot can navigate to the user and provide assistance in poor IAQ scenarios using the Kinect camera. Moreover, the system is able to communicate; it triggers alerts automatically using the Facebook social network if needed. The iAirBot system aims to reduce response time in the detection of poor IAQ conditions. It provides information access to the caregiver using the Facebook social network to respond in a timely manner for enhanced living environments and occupational health.

The rest of this paper is structured as follows: Section 2 focuses on the related work, Section 3 describe the methods and materials used in the implementation of the proposed architecture, Section 4 presents the experimental results, and the conclusion is presented in Section 5.

\section{Related Work}

IoT and AAL technologies present relevant approaches for developing enhanced solutions for our day to day activities in numerous domains for enhanced living environments and well-being. New methods and technologies are being reported to increase the number of intelligent cyber-physical systems that can be installed in regular buildings. These two closely related fields of research provide an effective base of knowledge for developing automatic solutions for enhanced living environments. To promote occupational health, the majority of living environments should be monitored in real-time and must incorporate enhanced methods for data access and to alert the user in case of poor ambient quality. On the one hand, on average, the population of developed countries spends about $90 \%$ of their time inside buildings. As such, IAQ monitoring is essential for creating improved living environments 
and for occupational health. On the other hand, the world population is ageing, which will lead to increased costs to maintain the quality of life of the elderly, including caregiver costs. Furthermore, buildings have several sources of pollutants. Therefore, the development of assistive robots for assisted living environments and indoor quality monitoring is particularly relevant. In this section, some studies related to $i$ AirBot are analyzed.

FLORENCE is a telepresence project based on the TurtleBot platform [24]. According to the author, the Skype application has good quality video and audio. This makes it possible to control the robot safely and it is user-friendly. The skype4py library is used to connect to the Skype application programming interface (API), which is linked to the application that allows remote control of the robot through Websockets.

The Domestic Robot for Elderly Assistance (DOMEO) project is a home care robot for the elderly [25]. It uses Microsoft speech recognition software. The following services are provided: reminders, games, shopping list, weather forecast, web browsing, video conferencing (using Skype), remote support, telepresence, following the user, and automatic upload.

A conceptual approach to the use of scalable robotic systems for the development of assisted living environments proposes the use of embedded systems of the Arduino platform and Ethernet technology [26]. They analyze scalability and modularity for this type of system. The idea will be to build an adaptive sensor network in any room, in which functionalities can be added according to the needs of each user. This concept shows that the idea that assisted living environments are only possible in smart homes is obsolete. This is a challenging interdisciplinary approach at design, social, and cultural levels.

The ASTRO project is an assistive robot for the elderly [27]. ASTRO aims to study the design, development, and testing of a robot that is integrated into intelligent environments. The robot would be able to perform basic tasks, such as helping an elderly person get up from a sofa or bed, access objects that are located in the robot, make telephone calls, access multimedia content, alert the user to take the medication and provide reminders, alert the elderly to some of the critical parameters of the home, and also includes telepresence. This robot uses a Zigbee sensor network to determine its location by measuring the received signal strength indication (RSSI) value.

A fall detection project, which uses a Microsoft Kinect device, is proposed in [28]. The goal is to detect falls in older adults and set subsequent warning actions to get quick assistance. This project studied the application of two different algorithms. The first uses a simple method for instantaneous detection, using only 1 frame of data. The second is more detailed because it tries to distinguish actual falls from an individual simple lying down. For falls, warnings can be sent via either SMS or e-mail, and may include images of the occurrence. This project also includes the possibility of cancellation of "false falls" by voice command.

The Autonomous Robot for Transport and Service (ARTOS) project developed a prototype of a completely autonomous assistive robot [29]. The localization, mapping, and navigation system is behavior-based. According to the author, this type of system allows effective and robust control of the navigation and location of the robot inside a house. Additionally, it uses laser sensors, ultrasound, and RFID sensor data distributed throughout the house to obtain a precise location.

RP-VITA is an intuitive and user-friendly remote presence robot [30]. It replaces the need for "telehealth" teams and can be used remotely with a mobile device. RP-VITA integrates 30 sensors for detection of obstacles and trajectory planning. A screen is at the top of the robot to enable users to interact with the service provider. These features contribute to simplifying time-consuming tasks that are faced by healthcare personnel. RP-VITA combines telecommunication capabilities and autodrive technology into an innovative, easy-to-use, independent telehealth solution to enable healthcare professionals to focus more on patients. InTouch Health and iRobot built this robot. RP-VITA moves independently without colliding with people and objects. It also has interfaces for connecting multiple sensors.

The "AssistiveAble" project has shown progress in the development of an assistive robot for a social assisted house environment [31]. This project focuses on older adults with mild cognitive 
impairment who live alone. The robot's range of functionalities is broad, including tasks related to taking medication, drinking, and cognitive stimulation. It is possible to videoconference with the robot; this feature enables the elderly to interact with staff, family, and service providers. This robot allows the detection and evaluation of critical situations, such as fall detection.

There are several IoT-based projects for IAQ monitoring that incorporate open source technologies for data processing and transmission and data acquisition microsensors, and also allow access to data collected from different sites simultaneously using mobile computing. These projects have been applied to several multidisciplinary research fields, such as medical applications [32] and agriculture [33]. A WSN approach to IAQ monitoring to improve the environmental quality of buildings for enhanced occupational health is proposed in [34]. A health informatics solution for IAQ that incorporates mobile computing technologies is proposed in [35]. This solution incorporates several sensors to monitor temperature, humidity, carbon monoxide (CO), carbon dioxide $\left(\mathrm{CO}_{2}\right)$, and light. An IoT architecture for indoor monitoring and control is proposed by [36]. This system is based on the Arduino platform; it incorporates low-cost sensors for data collection. Data access is performed using desktop, web, and mobile applications.

Numerous IoT architectures have been developed for monitoring, using not only specific IAQ parameters such as particulate matter (PM) [37-39] and $\mathrm{CO}_{2}$ [40], but also multi-gas sensors [41,42]. A context-aware mobile sensing system for indoor environment monitoring is proposed by [43]. This system is based on the Arduino platform and incorporates temperature, humidity, $\mathrm{CO}$, and $\mathrm{CO}_{2}$ sensors. The data is collected and uploaded to a server using Wi-Fi communication technologies, but also to a mobile phone using Bluetooth Low Energy (BLE) technology.

Another approach using autonomous indoor robots to supervise occupant comfort and energy parameters inside buildings is proposed by [44]. This system is based on the TurtleBot for navigation and incorporates several sensors to monitor several parameters, such as temperature, humidity, $\mathrm{CO}_{2}$, light, occupancy levels, airspeed, and electricity consumption. An IAQ monitoring system based on a hybrid WSN-IoT architecture is proposed by [45]. The system is composed of several sensor nodes that incorporate $\mathrm{CO}_{2}$ sensors and upload the IAQ information to a remote server for data visualization. An indoor autonomous mobile robot system for environmental quality supervision enabled by a sensor-rich navigation-capable robot to actively survey the indoor space is proposed by [46]. The proposed automated mobile system incorporates $\mathrm{CO}_{2}$, light, volatile organic compounds (VOC), and temperature sensors; it uses Wi-Fi communication technology. The open-source smart lamp project proposes a smart object for indoor environmental quality management based on the Arduino platform [47]. This system incorporates temperature, humidity, $\mathrm{CO}_{2}$, and a light sensor, and is a cost-effective solution to control indoor thermal comfort quality, IAQ, and indoor lighting quality.

A set of mobile robots connected via Wi-Fi to a cloud server to provide environmental supervision is proposed by [48]. The master robot is configured as a base station, while the others are configured as sensor nodes that are responsible for the data acquisition and for transmitting the collected data to the base station. The base station receives the data and uploads it to the cloud service for analytics and visualization.

A WSN approach for IAQ monitoring based on the Waspmote platform using ZigBee communication technology for data transmission is proposed by [49]. The sensor nodes have low-cost temperature, humidity, $\mathrm{CO}$, and $\mathrm{CO}_{2}$ sensors for data acquisition, but also professional equipment for PM supervision, namely DUSTTRAK DRX 8533 Aerosol (TSI, Shoreview, MN, USA). The collected data can be viewed using a desktop application developed in Java. The base station receives IAQ data from several indoor locations sent by the sensor's nodes.

In summary, several assistive robots are described above, but none of the reviewed research projects incorporate IAQ monitoring and social network integration. Moreover, the IoT and WSN projects for IAQ monitoring do not provide integration with assistive robots. Therefore, the iAirBot is a significant contribution to actual IAQ monitoring systems and assistive robots. 


\section{Materials and Methods}

The iAirBot is an assistive robot for IAQ monitoring systems based on IoT that works in real-time. It is based on TurtleBot 2, which is a low-cost kit used for developing assistive robots with open-source software. Figure 2 illustrates the iAirBot system. It incorporates a WSN, the sensor node is called the iAirNode Sensor, and the central node or concentrator is called the iAirNode Gateway.

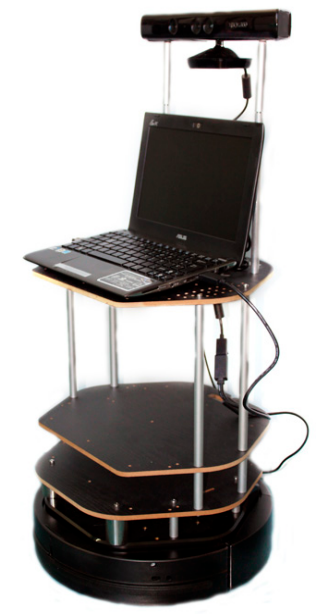

Figure 2. The iAirBot system hardware.

The parameters are monitored using the iAirNode Sensor, which collects air quality information and sends it to the iAirNode Gateway system. The iAirNode Gateway is connected via a USB interface to the iAirBot computer. The iAirBot is responsible for the interface with the social network Facebook for the sharing of the data monitored using web services. These web services are developed in ASP.NET and implement the Facebook API software development kit (SDK). This API enables access to the page where the monitored data is published. The platform used to create the sensor network is called Sun Small Programmable Object Technology (Sun SPOT). These sensor nodes are responsible for acquiring data and sending it to the base station via radio communication. The sensor network is the result of a large number of small-scale nodes with data collection capabilities organized in a cooperative network [50]. Sensor networks are optimized through wireless communication to provide continuous monitoring of the environmental conditions surrounding the nodes. They incorporate low energy consumption at each node. Wireless communication is implemented using the Sun SPOT module, which uses the IEEE 802.15.4 network protocol and supports the Zigbee protocol [51]. The IEEE 802.15.4 standard specifies the access control layers for wireless communication in private areas.

Communication between the iAirNode Gateway and the iAirNode Sensor takes place via radio, where the iAirNode Gateway is always listening for packets of information [52]. The communication architecture between iAirNode Sensor, iAirNode Gateway, and the iAirBot is illustrated in Figure 3.

The iAirBot incorporates a Kinect camera to browse, locate, and track people in indoor environments. FreeTTS was implemented to provide voice synthesis to the system. FreeTTS is a voice synthesis system developed in Java programming language. CMU Sphinx was implemented to provide voice recognition. CMU Sphinx is one of the voice recognition systems developed at Carnegie Mellon University. These systems include voice recognition and an acoustic instructor (SphinxTrain). Sphinx is an open-source voice recognizer that brings a dictionary that restricts word synthesis [53]. 


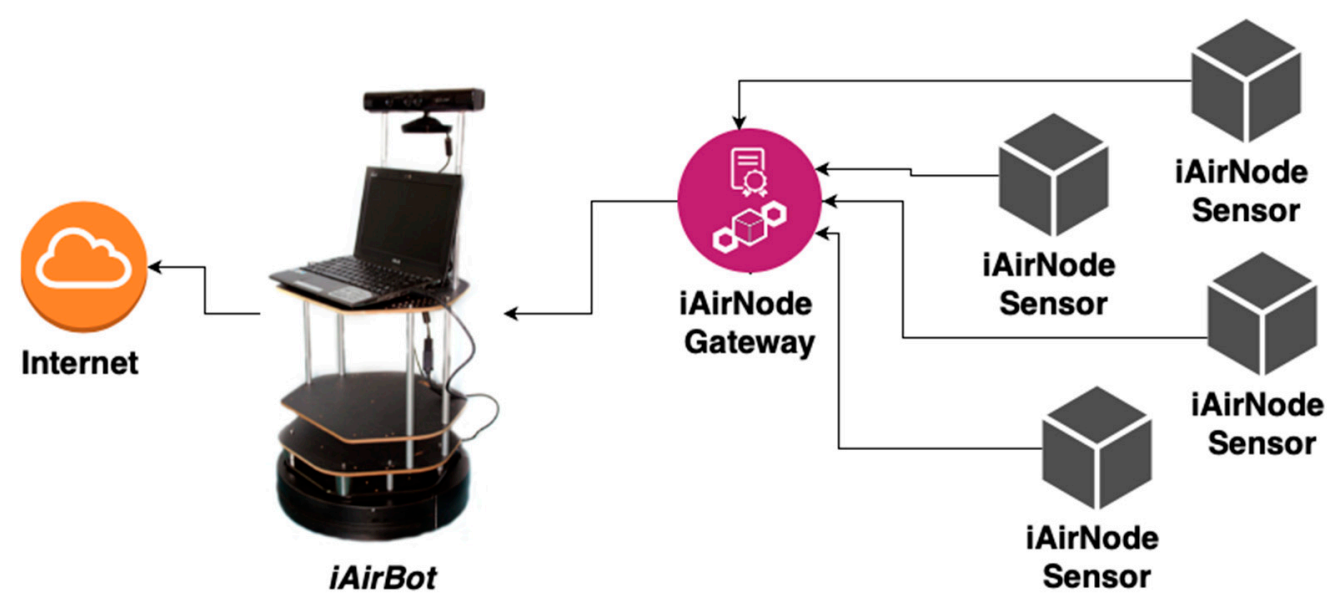

Figure 3. The iAirNode Sensor, iAirNode Gateway, and iAirBot communication diagram.

When the sensor output reaches a threshold where poor IAQ conditions are detected, the iAirBot navigates to the user and asks them to ask for help. If the user does not answer in $30 \mathrm{~s}$, the robot automatically launches an alert to the social network Facebook (accessed by the caregivers and relatives of the elderly). On the other hand, if the user responds positively to the robot saying, "Yes I need help", the robot proceeds in the same way by creating a post on the Facebook network. When the robot detects that the individual responds, "I do not need", the robot asks again by saying, "Did you say that you do not need help?". The individual must confirm again that they do not need help. In this case, the robot does not publish any information on the social network. The individual detection and navigation module is integrated directly with the Kinect class, which is responsible for the treatment of camera events. In summary, the proposed system provides real-time alerts using the Facebook social network but also directly interacts with the individual using a pervasive and ubiquitous approach.

Figure 4 shows the main modules used in the iAirBot. These are the Kinect camera (Kinect class), speech recognition and synthesizer software (based on the Free TTS and Sphinx software, respectively), an emergency warning module that posts messages on the social network Facebook, and a sensor network to monitor the indoor environment.

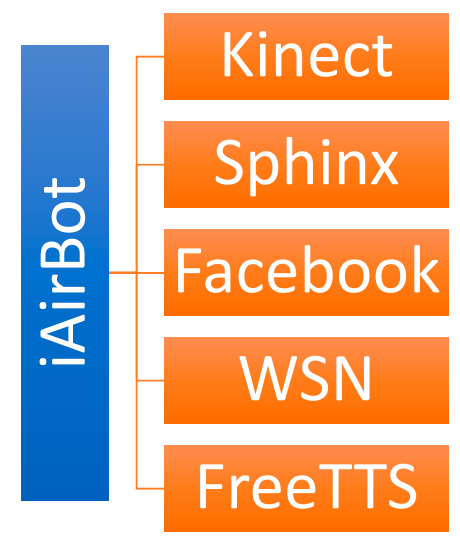

Figure 4. The iAirBot modules. Note: $\mathrm{WSN}=$ wireless sensor network.

Figure 5 shows the architecture of the IAQ monitoring system. It shows the various connections between the Sun SPOT nodes and the gas sensor, as well as the Internet connection. The MQ-6 sensor is connected to the iAirNode Sensor via an I2C connection. The Sun SPOT module that receives the data is connected to the robot, which in turn is wirelessly connected to the Internet so that it can publish on Facebook. 
IEEE 802.15.4

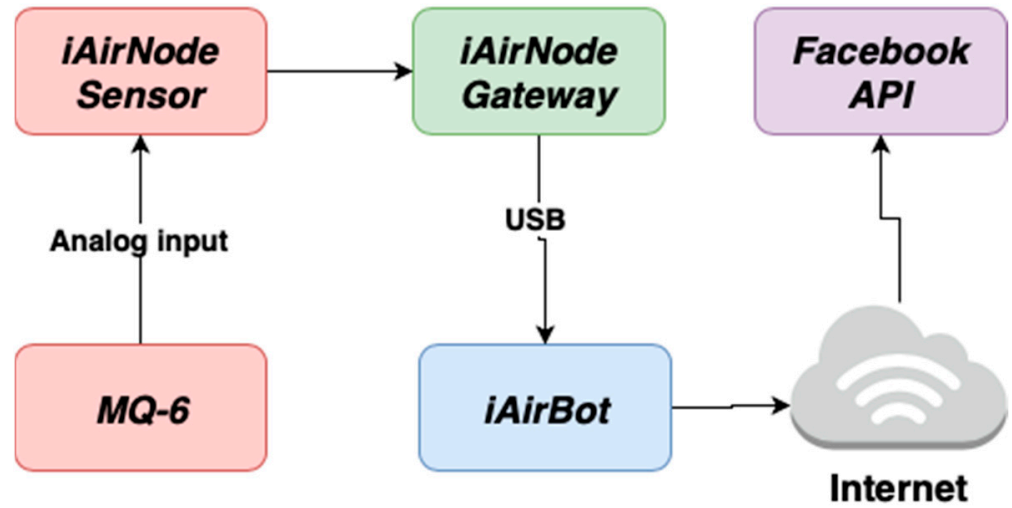

Figure 5. Indoor air quality (IAQ) monitoring architecture.

The MQ-6 sensor (Zhengzhou Winsen Electronics Technology Co., Ltd., Zhengzhou, China) is highly sensitive to LPG iso-butane and propane, and has slight sensitivity to alcohol and smoke. This sensor has a fast response and provides stable output measurements. The concentration range is 300-10,000 ppm (Propane). Figure 6 presents the propane sensitivity curve [54]. Furthermore, the sensor is used and validated in numerous research studies, particularly in the AAL field [55-58]. This sensor presents a reliable and cost-effective option for LPG supervision. However, other sensors with more accuracy are available on the market but at a higher cost.

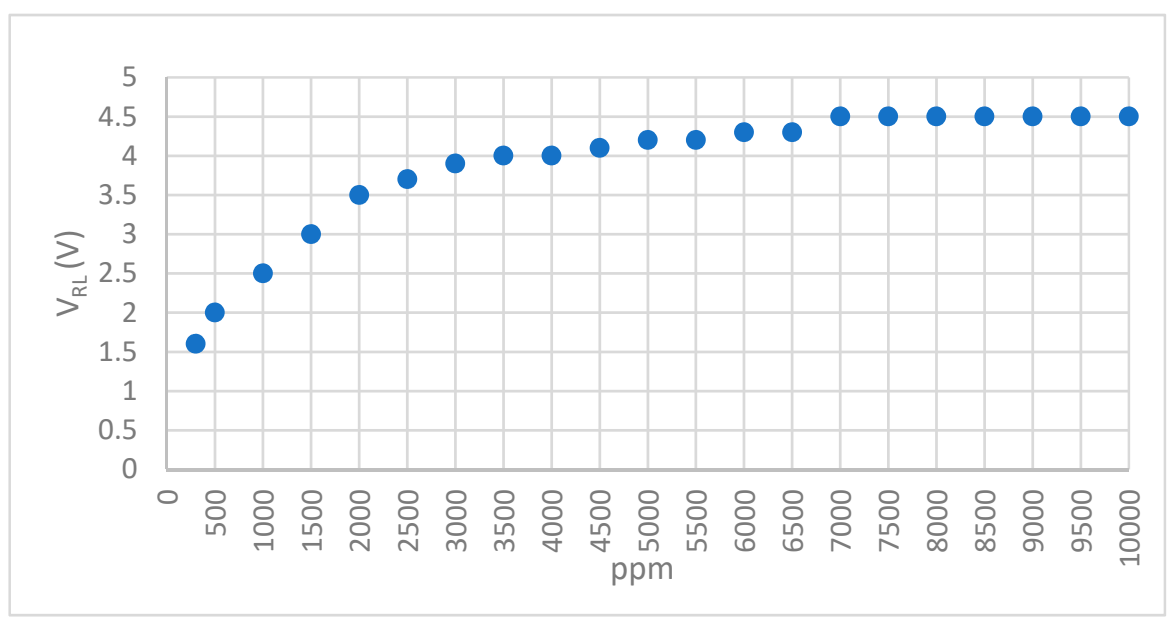

Figure 6. MQ-6 sensitivity curve with different concentrations of propane.

The proposed architecture is based on the Facebook API for data sharing and notifications. To provide secure access to Facebook data, a Facebook application was developed. The Facebook application has several properties, such as an application name, namespace, and category. A NET web service was created for data exchange and interaction. Moreover, the Facebook application ID and application secret need to be saved, as these properties are required to configure the web service. This web service implements the Facebook .NET SDK to read and write data to a Facebook page. The data collected by the iAirNode Sensor is transmitted to the AirNode Gateway, which is connected to the AirBot. The AirNode Gateway is responsible for making the web service calls that publish data on the Facebook page. The web methods are designed to create posts on the social network Facebook; these methods take a string of text to publish and create a post on the page showing the user, which is specified by the token; it then returns a success message. To start using this system at the first stage, the user is redirected to a web page, where they must accept the application permissions. In this case, after obtaining the token, the application can publish on the users page. Furthermore, the proposed 
architecture connects different components as a bridge between the IAQ, WSN, and social networks, using an IoT approach and assistive robots (Figure 7).

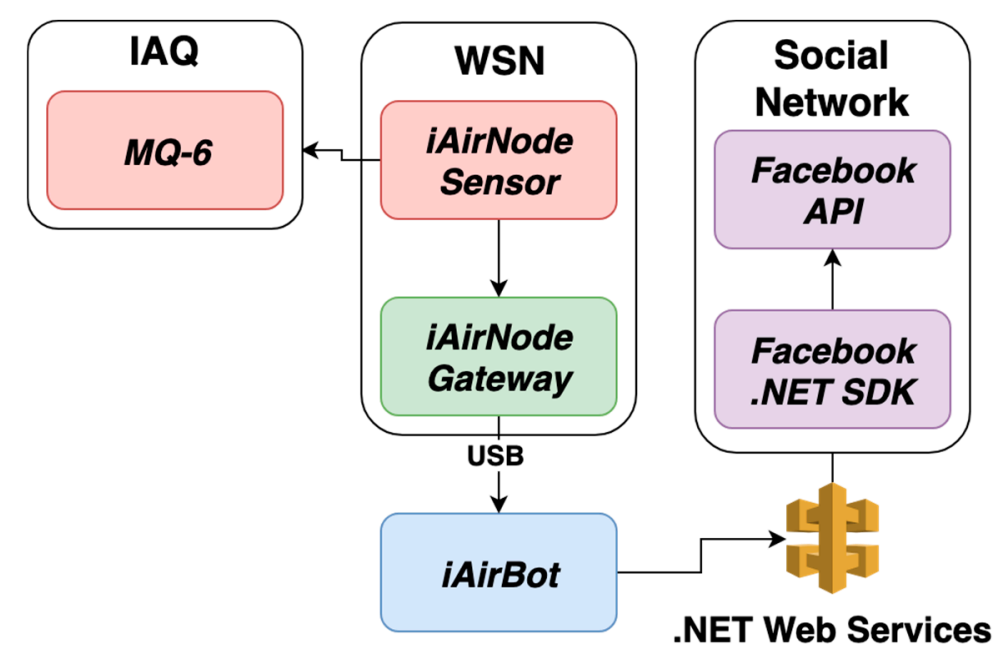

Figure 7. IAQ, WSN, and social network integration architecture.

In the iAirBot project, FreeTTS is used to send alerts and questions to the elderly. When the user answers to the robot, it recognizes the voice based on the defined grammar. Regarding the numerous cases of explosions and intoxication caused by LPG, which mainly affects older people who use this type of energy for cooking activities and heating systems, the use of iAirBot is proposed by the authors. For IAQ monitoring, the iAirBot incorporates an MQ-6 gas sensor connected to the iAirNode Sensor's analogue input. The proposed system is a case study for the previously described problem (Figure 8). An MQ-6 gas sensor was selected to provide a cost-effective solution, but also as the main objective of this study was to test the functional architecture of the solution to address this specific scenario. The proposed architecture aims to improve household safety for older adults to help them remain living in their own homes for as long as possible. Other sensors can be added to monitoring specific IAQ pollutants, such as $\mathrm{CO}_{2}, \mathrm{PM}$, and $\mathrm{CO}$, but also for supervision of thermal comfort parameters, such as temperature and humidity.

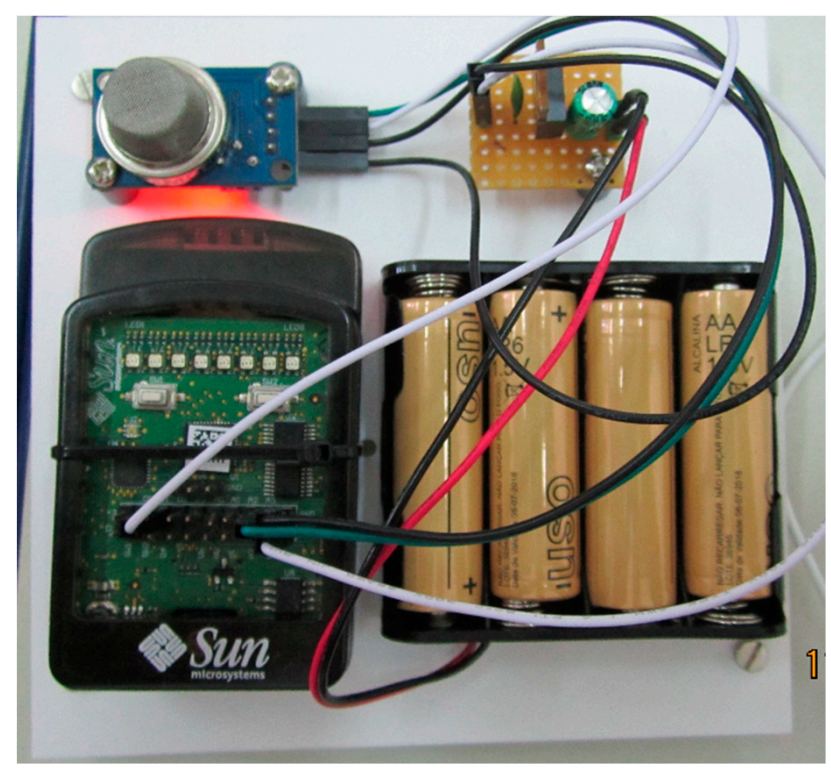

Figure 8. The iAirNode Sensor hardware. 
At startup, the iAirNode Sensor opens a radio connection to the iAirNode Gateway, which is connected to the iAirBot. The iAirNode Sensor verifies the IAQ every $100 \mathrm{~ms}$. If the setpoint set is exceeded, this means there is gas in the room, and a notification is sent to the iAirNode Gateway (Figure 9a).

In turn, the iAirNode Gateway is connected via USB to the iAirBot and communicates with this through the serial port. The iAirNode Gateway listens to messages sent via radio to port 70 . All information received via the iAirNode Gateway's radio is sent from the USB port to the iAirBot.

The iAirNode Gateway checks every $500 \mathrm{~ms}$ to see if there are any data packets from any iAirNode Sensors (Figure 9b).

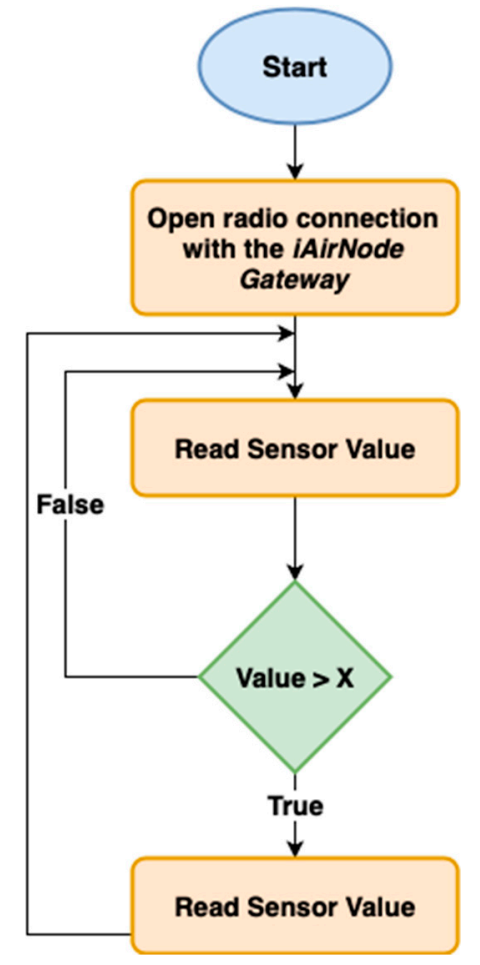

(a)

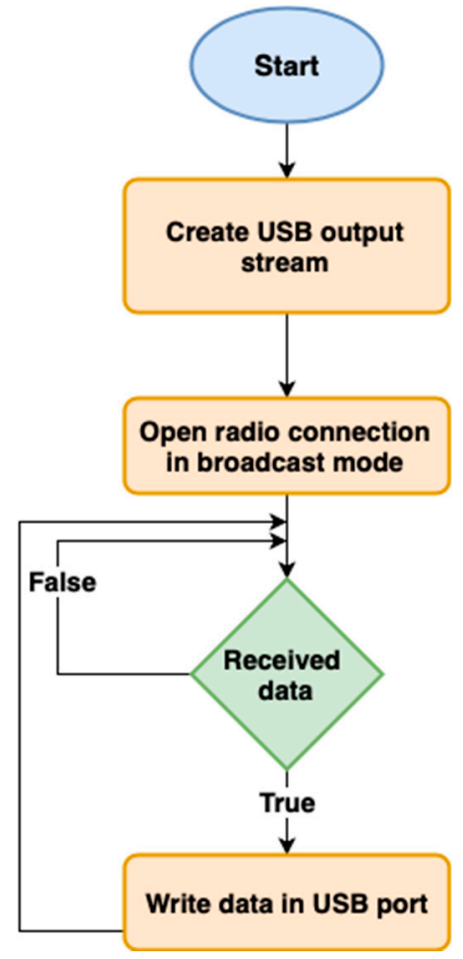

(b)

Figure 9. WSN architecture: (a) iAirNode Sensor architecture; (b) iAirNode Gateway architecture.

The fact that the iAirNode Gateway broadcasts rather than a specific iAirNode Sensor enables the use of multiple iAirNode Sensor that can be placed in multiple locations. In turn, this allows system modularity and scalability. The application runs on iAirBot for management of data that is sent by the iAirNode Gateway via USB, and was implemented using the Java language. When an alert is received, it is published on the Facebook social network, and iAirBot passes this on to the user (with a predefined movement) to alert them to what has happened by saying, "indoor air quality warning".

\section{Discussion and Results}

Dwelling, construction, heating, and ventilation types affect IAQ. It is estimated that two-thirds of indoor environments with natural ventilation are particularly airtight, and the remaining third tend to be leakier [59]. The RSSI variation related to the distance between nodes was tested to ensure the system resistance to interference. RSSI measures the relative signal quality received on a device. Each RSSI test consisted of sending 500 packets, and the average RSSI was calculated. RSSI tests were conducted in building corridors. The objective of RSSI tests was to measure the maximum distance between nodes to ensure the effective application of the proposed architecture in a real environment. Moreover, the conducted tests considered the WSN nodes positioned on the ground and at $1 \mathrm{~m}$ in 
height, with and without amplification. The IAQ monitoring tests were conducted inside a laboratory of a Portuguese university, which was monitored on-site using the iAirBot.

Figure 10 represents the monitored laboratory and corridor $(2.04 \mathrm{~m} \times 48.58 \mathrm{~m})$ scheme where the authors performed the experiments. The system was placed inside the building to monitor the laboratory environment (identified with "B" in Figure 10), and the sensor module was placed in the center of the room (identified with " $x$ "). As in most indoor environments, the space monitored was naturally ventilated, without any dedicated ventilation slots on the facades. Natural ventilation is performed through opening doors and windows.

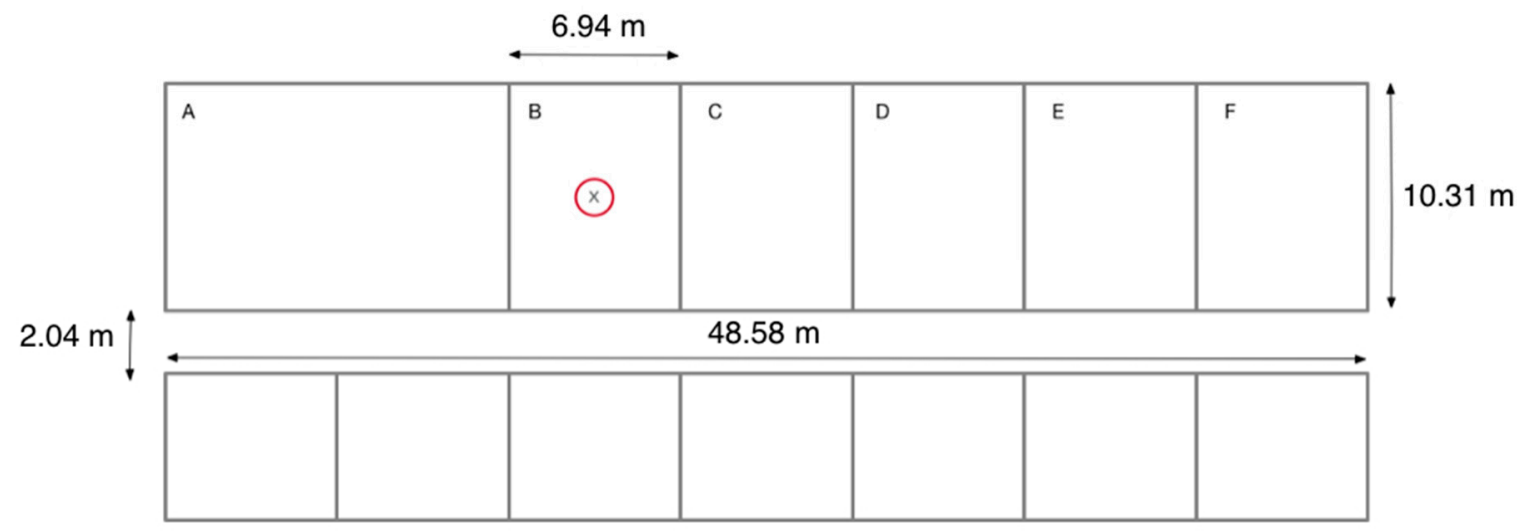

Figure 10. Scheme of the locations where the tests were conducted.

Figures 11-13 show the variation of the RSSI values. RSSI values were measured at floor level and up to $1 \mathrm{~m}$ in height, as well as with signal amplification.

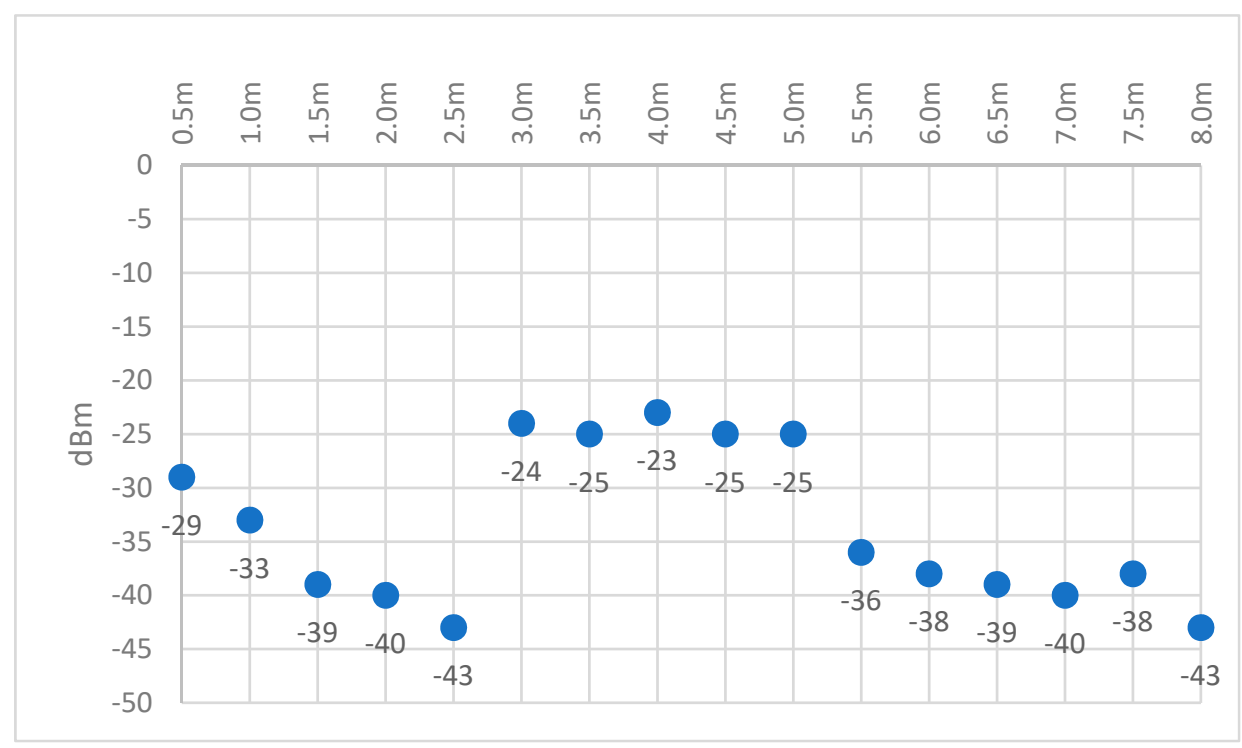

Figure 11. Variation of RSSI values measured at floor level. 


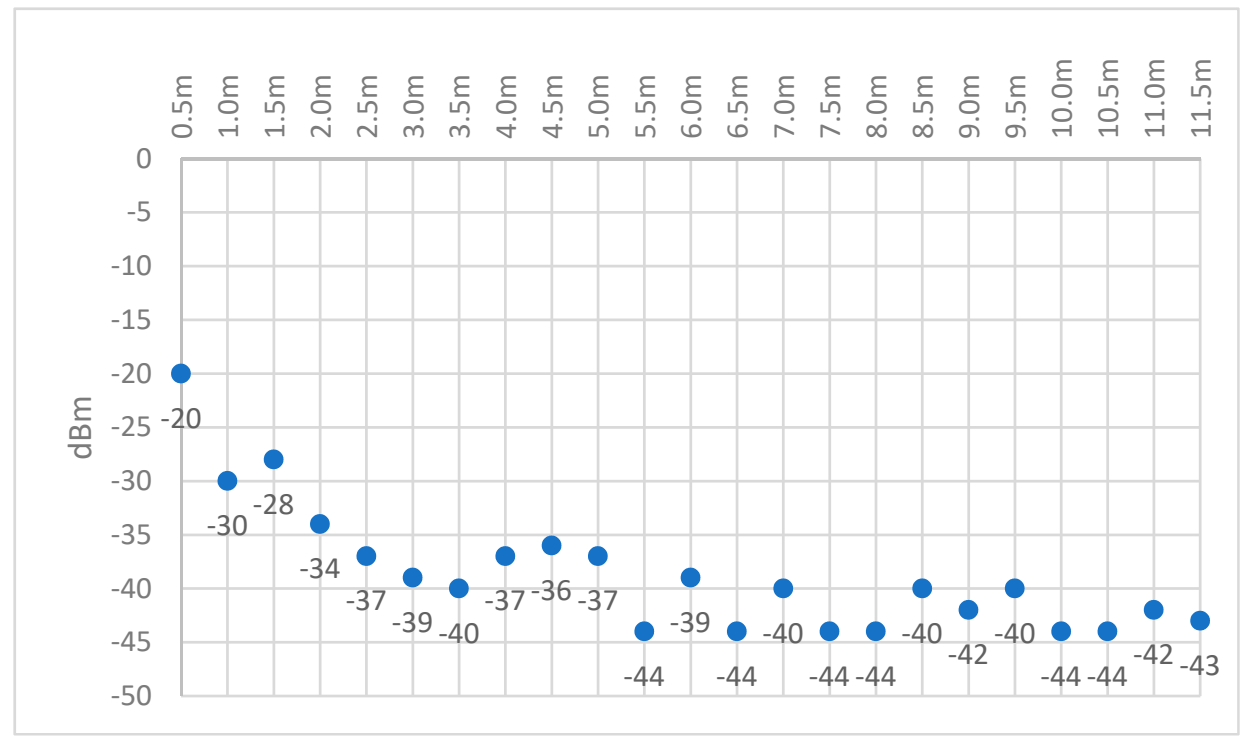

Figure 12. Variation of RSSI values measured at $1 \mathrm{~m}$ in height.

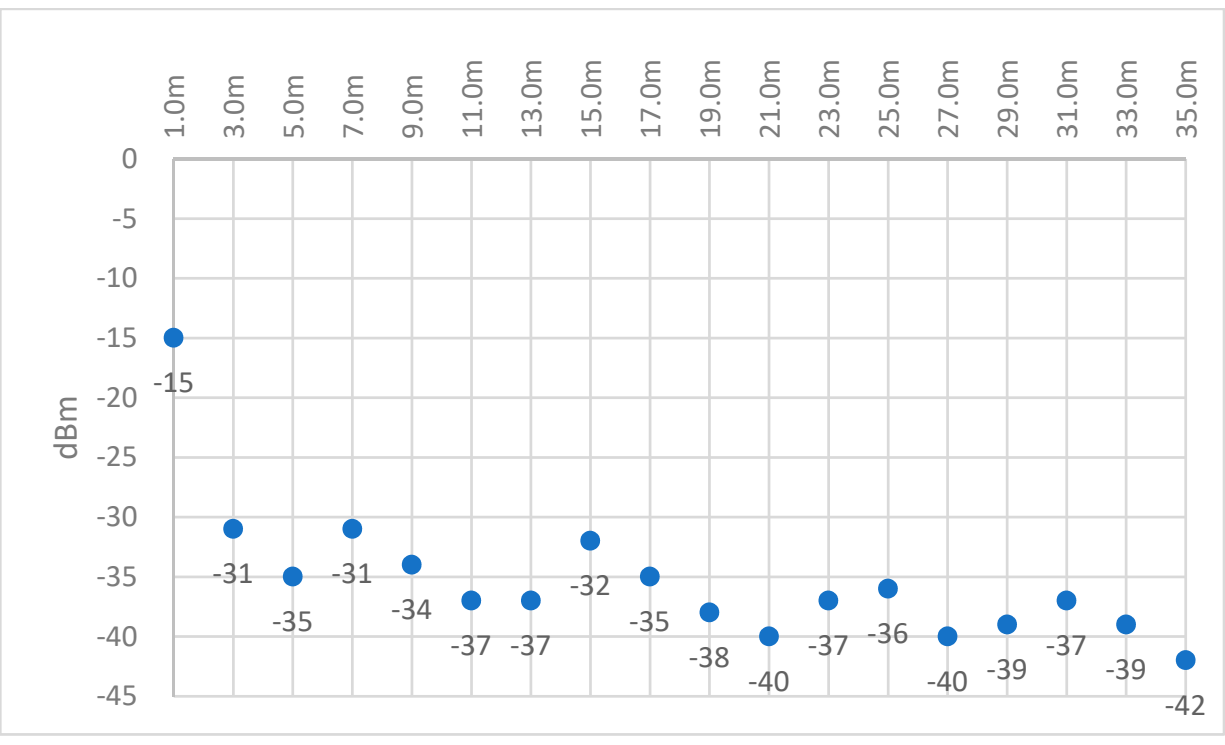

Figure 13. Variation of RSSI values measured at $1 \mathrm{~m}$ in height with signal amplification.

The RSSI test at floor level showed that the maximum distance between nodes was $8 \mathrm{~m}$. The RSSI test at $1 \mathrm{~m}$ height reported 3 to $5 \mathrm{~m}$ distance. The RSSI tests conducted at $1 \mathrm{~m}$ in height without amplification returned a maximum distance range of $11.5 \mathrm{~m}$ between nodes, which satisfies the requirements of most buildings. The RSSI tests verified that the product is feasible for implementation indoors when the nodes of the sensor network are placed at $1 \mathrm{~m}$ height. However, for larger interior dimensions, the system requires the use of signal amplification, as the maximum distance between nodes is up to $34 \mathrm{~m}$. The monitoring tests show that the proposed system can be used to detect poor IAQ at low-cost. Furthermore, the proposed system can provide an efficient and effective monitoring system for enhanced living environments and occupational health.

IAQ monitoring is a trending topic for which some other low-cost and open-source monitoring systems have been developed [43-49]. Due to the quality and relevant contribution of these solutions, a brief summary of these studies is presented in Table 1. 
Table 1. A review of similar types of IAQ supervision systems.

\begin{tabular}{|c|c|c|c|c|c|c|c|}
\hline & Controller & Sensors & Architecture & Open Source & Connectivity & $\begin{array}{c}\text { Automated } \\
\text { Mobile System }\end{array}$ & Social Networks \\
\hline $\begin{array}{l}\text { Lohani and Acharya } \\
\text { [43] }\end{array}$ & Arduino, ESP8266 & $\begin{array}{c}\text { Temperature, Relative } \\
\text { Humidity, } \mathrm{CO}_{2}\end{array}$ & IoT & $\sqrt{ }$ & Wi-Fi, BLE & $x$ & $\times$ \\
\hline Mantha et al. [44] & Netbook & $\begin{array}{c}\text { Temperature, Relative } \\
\text { Humidity, } \mathrm{CO}_{2} \text {, Light, } \\
\text { Occupancy Levels, } \\
\text { Airspeed and Electricity } \\
\text { Consumption }\end{array}$ & Local & $\times$ & $\times$ & $\sqrt{ }$ & $\times$ \\
\hline $\begin{array}{l}\text { Srivatsa and } \\
\text { Pandhare [45] }\end{array}$ & Raspberry Pi & $\mathrm{CO}_{2}$ & WSN/IoT & $\sqrt{ }$ & Wi-Fi & $x$ & $\times$ \\
\hline Jin et al. [46] & Arduino & $\begin{array}{l}\mathrm{CO}_{2} \text {, Light, VOC, } \\
\text { Temperature }\end{array}$ & IoT & $\sqrt{ }$ & Wi-Fi & $\sqrt{ }$ & $\times$ \\
\hline Salamone et al. [47] & Arduino & $\mathrm{CO}_{2}$ & WSN & $\sqrt{ }$ & Zigbee & $x$ & $\times$ \\
\hline Menna et al. [48] & Arduino & $\begin{array}{c}\text { Multi-gas Sensor, } \\
\text { Temperature, Relative } \\
\text { Humidity }\end{array}$ & WSN/IoT & $\sqrt{ }$ & Wi-Fi, Bluetooth & $\sqrt{ }$ & $\times$ \\
\hline Bhattacharyet al. [49] & Waspmote & $\begin{array}{l}\mathrm{CO}, \mathrm{CO}_{2}, \mathrm{PM}, \text { Temperature, } \\
\text { Relative Humidity }\end{array}$ & WSN & $\sqrt{ }$ & Zigbee & $x$ & $\times$ \\
\hline
\end{tabular}

Note: $\sqrt{ }=$ support; $\times$ = no support; $\mathrm{CO}_{2}=$ carbon dioxide; $\mathrm{VOC}=$ volatile organic compounds; $\mathrm{CO}=$ carbon monoxide; $\mathrm{PM}=$ particulate matter; $\mathrm{BLE}=\mathrm{Bluetooth}$ Low Energy. 
Compared to most of the assistive robots, iAirBot stands out for its modularity and the scalability of its sensors network, as well as the use of social networks for information sharing. The iAirBot offers social network integration, which is not provided by any of the systems described in Table 1 . On the one hand, Facebook has changed the nature of communication between people and can be assumed to be an efficient methodology to connect people in real-time. Facebook users have been increasing in number over time. On the other hand, Facebook provides a secure API for integration of third-party applications. Furthermore, this social network can be configured to provide an effective method to share data between people and theirs caregiver in real-time, helping them maintain security and privacy. For occupants without a Facebook account, the caregiver can use their own account for the Facebook application configuration. The system architecture is based on Facebook for data storage and notification features. Therefore, a Facebook account is mandatory for the configuration of the proposed architecture. In the future, it is planned to create a web application to provide alternative access for people that do not have any social media accounts.

Compared to other solutions proposed by the authors of $[43,45,47,49]$, which do not support automated mobile capabilities, the iAirBot provides an AAL approach for air quality monitoring. The proposed system is an assistive robot developed on top of the TurtleBot platform, which incorporates a WSN for environmental monitoring and a Kinect camera for navigation and user detection. The iAirBot adds IAQ monitoring features to standard functionalities for AAL, such as ubiquitous presence over the Internet and fall detection. Moreover, this system can be assumed as an effective method for incorporating air quality monitoring, which is particularly important for the elderly as they are most fragile. It also provide a pervasive method for data sharing with caregivers in real-time. The iAirBot propose a ubiquitous method to notify caregiver using social networks, which provide timely information that can be used to improve IAQ by providing ventilation or activation of air purification systems.

Numerous cases have been reported about explosions or intoxication caused by LPG, iso-butane, or propane air contamination, particularly with older people who forget to properly shut down systems that use this type of energy for cooking activities. Therefore, the first step is to provide IAQ monitoring and control for enhanced occupational health and well-being. The authors selected an LPG, iso-butane, and propane sensor to test the functional architecture. In order to prevent fires and explosions, the iAirNode Sensor is configured to detect low gas levels that are able to form explosive atmospheres. In the future, technical improvements and special precautions are planned to prepare the system to operate in potentially explosive atmospheres, implementing supporting resource command features to automatically switch off any fuel source in real-time. However, other sensors can be added to the $i$ AirBot to provide real-time monitoring of specific IAQ parameters. Numerous reliable, low-cost, and miniaturized sensors are available on the market, particularly for $\mathrm{CO}_{2}, \mathrm{PM}$, and $\mathrm{CO}$ monitoring. The iAirNode Sensor is compatible with all sensors described in Table 1. Furthermore, the authors plan to adapt and install the iAirBot system in specific fields, such as hospitals and elderly homes, for testing and validation purposes.

The iAirBot presents a significant method of detection that can alert caregivers in real-time to avoid events that may lead to the death of the occupants. The results are very important, as the proposed system can be used as a pervasive assistive robot solution, incorporating IAQ monitoring features at low cost. Further, this system has advantages in terms of modularity and scalability. The system can be installed with only one iAirNode Sensor, and according to the complexity of the design, more data acquisition sensors can be added. However, it should be noted that the iAirBot is still in a testing phase, and calibration improvements are necessary.

In the future, it is planned to make hardware and software improvements regarding the notification methods. Moreover, the authors also plan to develop a mobile application for data consultation and analysis. 


\section{Conclusions}

The iAirBot is an assistive robot that incorporates IAQ monitoring solutions based on IoT architectures for enhanced living environments. The proposed system includes WSN for environmental monitoring and automated capabilities that provide data sharing via the Facebook social network. The results show that the proposed cost-effective system stands out because of its use of a social network for information sharing. Furthermore, this system has advantages in terms of modularity and scalability, as the installation can start by using only one sensor node, and according to the complexity of the design, more data acquisition sensors can be added. Facebook is an effective method for sharing data between elderly people and their caregiver in real-time, while maintaining security and privacy. The iAirBot adds IAQ monitoring features to standard functionalities for AAL, providing a ubiquitous presence over the Internet and fall detection. Using the $i$ AirBot, it is possible to detect and prevent explosions or intoxication scenarios caused by LPG, iso-butane, or propane air contamination, and notify the caregiver in real-time. However, the proposed system has some limitations, as the iAirBot needs further experimental validation for better calibration and accuracy. Several technical improvements are planned, such as the development of other alerting methods, such as SMS or e-mail, to advise the user when IAQ reaches certain values, and a mobile application for data consultation and notifications. In the near future, assistive robots will incorporate IAQ monitoring features to store historical data for sharing with caregivers, as well as providing real-time alerts through social networks to reduce response time in the case of an accident.

Author Contributions: Conceptualization, methodology, software, validation, formal analysis, investigation, writing —original draft preparation, writing—review and editing: G.M., I.M.P. and N.M.; supervision: R.P.

Funding: This research received no external funding.

Acknowledgments: The financial support from the Research Unit for Inland Development of the Polytechnic Institute of Guarda is acknowledged. This work is funded by FCT/MEC through national funds and when applicable co-funded by FEDER-PT2020 partnership agreement under the project UID/EEA/50008/2019 (Este trabalho é financiado pela FCT/MEC através de fundos nacionais e quando aplicável cofinanciado pelo FEDER, no âmbito do Acordo de Parceria PT2020 no âmbito do projeto UID/EEA/50008/2019).

Conflicts of Interest: The authors declare no conflict of interest.

\section{References}

1. McNicoll, G. World population ageing, 1950-2050. Popul. Dev. Rev. 2002, 28, 814-816.

2. Euromonitor International. The Top 5 Oldest Countries in the World. 2019. Available online: https://blog. euromonitor.com/the-top-5-oldest-countries-in-the-world/ (accessed on 30 April 2019).

3. Kana, M.A.; Correia, S.; Peleteiro, B.; Severo, M.; Barros, H. Impact of the global financial crisis on low birth weight in Portugal: A time-trend analysis. BMJ Glob. Health 2017, 2, e000147. [CrossRef] [PubMed]

4. Correia, S.; Rodrigues, T.; Montenegro, N.; Barros, H. Critical evaluation of national vital statistics: The case of preterm birth trends in Portugal. Acta Obstet. Gynecol. Scand. 2015, 94, 1215-1222. [CrossRef] [PubMed]

5. PORDATA. Índice de Dependência de Idosos: Que Países têm Mais e Menos Idosos por 100 Pessoas em Idade Activa? 2018. Available online: https://www.pordata.pt/Europa/\%C3\%8Dndice+de+depend\%C3\% AAncia+de+idosos-1929 (accessed on 27 October 2019).

6. Colantonio, S.; Coppini, G.; Giorgi, D.; Morales, M.-A.; Pascali, M.A. Computer Vision for Ambient Assisted Living. Comput. Vis. Assist. Healthc. 2018, 147-182. [CrossRef]

7. Marques, G. Ambient Assisted Living and Internet of Things. Harnessing the Internet of Everything (IoE) for Accelerated Innovation Opportunities; Cardoso, P.J.S., Monteiro, J., Semião, J., Rodrigues, J.M.F., Eds.; IGI Global: Hershey, PA, USA, 2019; pp. 100-115.

8. Marques, G.; Pitarma, R.; Garcia, N.M.; Pombo, N. Internet of Things Architectures, Technologies, Applications, Challenges, and Future Directions for Enhanced Living Environments and Healthcare Systems: A Review. Electronics 2019, 8, 1081. [CrossRef] 
9. Koleva, P.; Tonchev, K.; Balabanov, G.; Manolova, A.; Poulkov, V. Challenges in designing and implementation of an effective Ambient Assisted Living system. In Proceedings of the 2015 12th International Conference on Telecommunication in Modern Satellite, Cable and Broadcasting Services (TELSIKS), Niš, Serbia, 14-17 October 2015; pp. 305-308.

10. Centers for Disease Control and Prevention. The State of Aging and Health in America 2007. 2007. Available online: https://www.cdc.gov/aging/pdf/saha_2007.pdf (accessed on 27 October 2019).

11. Walsh, P.J.; Dudney, C.S.; Copenhaver, E.D. Indoor Air Quality; CRC Press: Boca Raton, FL, USA, 1983.

12. Cetin, M.; Sevik, H. Measuring the Impact of Selected Plants on Indoor CO2 Concentrations. Pol. J. Environ. Stud. 2016, 25, 973-979. [CrossRef]

13. Bruce, N.; Perez-Padilla, R.; Albalak, R. Indoor air pollution in developing countries: A major environmental and public health challenge. Bull. World Health Organ. 2000, 78, 1078-1092.

14. Seguel, J.M.; Merrill, R.; Seguel, D.; Campagna, A.C. Indoor Air Quality. Am. J. Lifestyle Med. 2017, 11, $284-295$. [CrossRef]

15. Butz, A.M. A Randomized Trial of Air Cleaners and a Health Coach to Improve Indoor Air Quality for Inner-City Children With Asthma and Secondhand Smoke Exposure. Arch. Pediatrics Adolesc. Med. 2011, 165, 741. [CrossRef]

16. De Vito, S.; Fattorusoa, G.; Liguoro, R.; Oliviero, A.; Masseraa, E.; Sansone, C.; Casola, V.; di Francia, G. Cooperative 3D Air Quality Assessment with Wireless Chemical Sensing Networks. Procedia Eng. 2011, 25, 84-87. [CrossRef]

17. Atzori, L.; Iera, A.; Morabito, G. The Internet of Things: A survey. Comput. Netw. 2010, 54, $2787-2805$. [CrossRef]

18. Xia, F.; Yang, L.T.; Wang, L.; Vinel, A. Internet of Things. Int. J. Commun. Syst. 2012, 25, 1101-1102. [CrossRef]

19. Pattar, S.; Buyya, R.; Venugopal, K.R.; Iyengar, S.S.; Patnaik, L.M. Searching for the IoT Resources: Fundamentals, Requirements, Comprehensive Review and Future Directions. IEEE Commun. Surv. Tutor. 2018, 20, 2101-2132. [CrossRef]

20. Agiwal, M.; Saxena, N.; Roy, A. Towards Connected Living: 5G Enabled Internet of Things (IoT). IETE Tech. Rev. 2018, 36, 190-202. [CrossRef]

21. Van den Dam, R. Internet of Things: The Foundational Infrastructure for a Smarter Planet. In Internet of Things, Smart Spaces, and Next Generation Networking; Balandin, S., Andreev, S., Koucheryavy, Y., Eds.; Springer: Berlin/Heidelberg, Germany, 2013; Volume 8121, pp. 1-12.

22. Bright, L.F.; Kleiser, S.B.; Grau, S.L. Too much Facebook? An exploratory examination of social media fatigue. Comput. Hum. Behav. 2015, 44, 148-155. [CrossRef]

23. Bayer, J.; Ellison, N.; Schoenebeck, S.; Brady, E.; Falk, E.B. Facebook in context(s): Measuring emotional responses across time and space. N. Media Soc. 2018, 20, 1047-1067. [CrossRef]

24. Sullivan, F.R. Robotics and science literacy: Thinking skills, science process skills and systems understanding. J. Res. Sci. Teach. 2008, 45, 373-394. [CrossRef]

25. Mayer, P.; Beck, C.; Panek, P. Examples of multimodal user interfaces for socially assistive robots in Ambient Assisted Living environments. In Proceedings of the 2012 IEEE 3rd International Conference on Cognitive Infocommunications (CogInfoCom), Kosice, Slovakia, 2-5 December 2012; pp. 401-406.

26. Kranz, M.; Linner, T.; Ellmann, B.; Bittner, A.; Roalter, L. Robotic service cores for ambient assisted living. In Proceedings of the 4th International ICST Conference on Pervasive Computing Technologies for Healthcare, Munchen, Germany, 22-25 March 2010.

27. Cavallo, F.; Aquilano, M.; Bonaccorsi, M.; Limosani, R.; Manzi, A.; Carrozza, M.C.; Dario, P. On the design, development and experimentation of the ASTRO assistive robot integrated in smart environments. In Proceedings of the 2013 IEEE International Conference on Robotics and Automation, Karlsruhe, Germany, 6-10 May 2013; pp. 4310-4315.

28. Kawatsu, C.; Li, J.; Chung, C.J. Development of a Fall Detection System with Microsoft Kinect. In Robot Intelligence Technology and Applications 2012; Kim, J.-H., Matson, E.T., Myung, H., Xu, P., Eds.; Springer: Berlin/Heidelberg, Germany, 2013; Volume 208, pp. 623-630.

29. Mehdi, S.A.; Armbrust, C.; Koch, J.; Berns, K. Methodology for robot mapping and navigation in assisted living environments. In Proceedings of the 2nd International Conference on PErvsive Technologies Related to Assistive Environments-PETRA '09, Corfu, Greece, 9-13 June 2009; pp. 1-6. 
30. Qureshi, M.O.; Syed, R.S. The Impact of Robotics on Employment and Motivation of Employees in the Service Sector, with Special Reference to Health Care. Saf. Health Work 2014, 5, 198-202. [CrossRef]

31. Suzuki, E.; Deguchi, Y.; Takayama, D.; Takano, S.; Scuturici, V.-M.; Petit, J.-M. Towards Facilitating the Development of Monitoring Systems with Low-Cost Autonomous Mobile Robots. In Information Search, Integration, and Personalization; Kawtrakul, A., Laurent, D., Spyratos, N., Tanaka, Y., Eds.; Springer International Publishing: Cham, Switzerland, 2014; Volume 421, pp. 57-70.

32. Feria, F.; Parra, O.J.S.; Daza, B.S.R. Design of an Architecture for Medical Applications in IoT. In Cooperative Design, Visualization, and Engineering; Luo, Y., Ed.; Springer International Publishing: Cham, Switzerland, 2016; Volume 9929, pp. 263-270.

33. Ray, P.P. Internet of things for smart agriculture: Technologies, practices and future direction. J. Ambient Intell. Smart Environ. 2017, 9, 395-420. [CrossRef]

34. Pitarma, R.; Marques, G.; Ferreira, B.R. Monitoring Indoor Air Quality for Enhanced Occupational Health. J. Med Syst. 2017, 41, 23. [CrossRef]

35. Marques, G.; Pitarma, R. Health informatics for indoor air quality monitoring. In Proceedings of the 2016 11th Iberian Conference on Information Systems and Technologies (CISTI), Las Palmas, Spain, 15-18 June 2016; pp. 1-6.

36. Marques, G.; Pitarma, R. Monitoring and control of the indoor environment. In Proceedings of the 201712 th Iberian Conference on Information Systems and Technologies (CISTI), Lisbon, Portugal, 21-24 June 2017; pp. 1-6.

37. Marques, G.; Ferreira, C.R.; Pitarma, R. A System Based on the Internet of Things for Real-Time Particle Monitoring in Buildings. Int. J. Environ. Res. Public Health 2018, 15, 821. [CrossRef] [PubMed]

38. Matz, J.R.; Wylie, S.; Kriesky, J. Participatory Air Monitoring in the Midst of Uncertainty: Residents' Experiences with the Speck Sensor. Engag. Sci. Technol. Soc. 2017, 3, 464. [CrossRef]

39. Marques, G.; Pitarma, R. mHealth: Indoor Environmental Quality Measuring System for Enhanced Health and Well-Being Based on Internet of Things. JSAN 2019, 8, 43. [CrossRef]

40. Marques, G.; Ferreira, C.R.; Pitarma, R. Indoor Air Quality Assessment Using a CO2 Monitoring System Based on Internet of Things. J. Med. Syst. 2019, 43, 67. [CrossRef] [PubMed]

41. Demuth, D.; Nuest, D.; Bröring, A.; Pebesma, E. The AirQuality SenseBox. In Proceedings of the EGU General Assembly Conference Abstracts, Vienna, Austria, 7-12 April 2013; Volume 15.

42. Marques, G.; Pitarma, R. A Cost-Effective Air Quality Supervision Solution for Enhanced Living Environments through the Internet of Things. Electronics 2019, 8, 170. [CrossRef]

43. Lohani, D.; Acharya, D. Smartvent: A context aware iot system to measure indoor air quality and ventilation rate. In Proceedings of the 2016 17th IEEE International Conference on Mobile Data Management (MDM), Porto, Portugal, 13-16 June 2016; Volume 2, pp. 64-69.

44. Mantha, B.R.; Feng, C.; Menassa, C.C.; Kamat, V.R. Real-time building energy and comfort parameter data collection using mobile indoor robots. In Proceedings of the ISARC 32nd International Symposium on Automation and Robotics in Construction, Oulu, Finland, 15-18 June 2015; Volume 32, pp. 1-9.

45. Srivatsa, P.; Pandhare, A. Indoor Air Quality: IoT Solution. In Proceedings of the National Conference NCPCI-2016, 19 March 2016; Volume 2016, p. 19.

46. Jin, M.; Liu, S.; Schiavon, S.; Spanos, C. Automated mobile sensing: Towards high-granularity agile indoor environmental quality monitoring. Build. Environ. 2018, 127, 268-276. [CrossRef]

47. Salamone, F.; Belussi, L.; Danza, L.; Galanos, T.; Ghellere, M.; Meroni, I. Design and Development of a Nearable Wireless System to Control Indoor Air Quality and Indoor Lighting Quality. Sensors 2017, 17, 1021. [CrossRef]

48. Meena, M.J.; Prabha, S.S.; Pandian, S. A cloud-based mobile robotic system for environmental monitoring. In Proceedings of the 2014 Asia-Pacific Conference on Computer Aided System Engineering (APCASE), South Kuta, Indonesia, 10-12 February 2014; pp. 122-126.

49. Bhattacharya, S.; Sridevi, S.; Pitchiah, R. Indoor air quality monitoring using wireless sensor network. In Proceedings of the 2012 Sixth International Conference on Sensing Technology (ICST), Kolkata, India, 18-21 December 2012; pp. 422-427.

50. Hill, J.; Horton, M.; Kling, R.; Krishnamurthy, L. The platforms enabling wireless sensor networks. Commun. ACM 2004, 47, 41. [CrossRef] 
51. Baig, F.; Mahmood, A.; Javaid, N.; Razzaq, S.; Khan, N.; Saleem, Z. Smart home energy management system for monitoring and scheduling of home appliances using zigbee. J. Basic. Appl. Sci. Res. 2013, 3, 880-891.

52. De Guglielmo, D.; Anastasi, G.; Seghetti, A. From IEEE 802.15.4 to IEEE 802.15.4e: A Step Towards the Internet of Things. In Advances onto the Internet of Things; Gaglio, S., Re, G.L., Eds.; Springer International Publishing: Cham, Switzerland, 2014; Volume 260, pp. 135-152.

53. Lamere, P.; Kwok, P.; Gouvea, E.; Raj, B.; Singh, R.; Walker, W.; Warmuth, M.; Wolf, P. The CMU SPHINX-4 speech recognition system. In Proceedings of the 2003 IEEE International Conference on Acoustics, Speech, and Signal Processing-ICASSP 2003, Hong Kong, China, 6-10 April 2003; Volume 1, pp. 2-5.

54. Zhengzhou Winsen Electronics Technology Co. Ltd. Flammable Gas Sensor (Model: MQ-6) Manual; Zhengzhou Winsen Electronics Technology Co. Ltd.: Zhengzhou, China, 2014.

55. Ilahi, N.I.; Baco, S.; Achmad, A.S.A.; Umrianah, E. Early Leakage Protection System of LPG (Liquefied Petroleum Gas) Based on ATMega 16 Microcontroller. IOP Conf. Ser. Mater. Sci. Eng. 2018, 336, 012021.

56. Ransing, R.S.; Rajput, M. Smart home for elderly care, based on Wireless Sensor Network. In Proceedings of the 2015 International Conference on Nascent Technologies in the Engineering Field (ICNTE), Navi Mumbai, India, 9-10 January 2015; pp. 1-5.

57. Gnanavel, R.; Anjana, P.; Nappinnai, K.S.; Sahari, N.P. Smart home system using a Wireless Sensor Network for elderly care. In Proceedings of the 2016 Second International Conference on Science Technology Engineering and Management (ICONSTEM), Chennai, India, 30-31 March 2016; pp. 51-55.

58. Keshamoni, K.; Hemanth, S. Smart Gas Level Monitoring, Booking \& Gas Leakage Detector over IoT. In Proceedings of the 2017 IEEE 7th International Advance Computing Conference (IACC), Hyderabad, India, 5-7 January 2017; pp. 330-332.

59. Persily, A.K. Field measurement of ventilation rates. Indoor Air 2016, 26, 97-111. [CrossRef] [PubMed]

(C) 2019 by the authors. Licensee MDPI, Basel, Switzerland. This article is an open access article distributed under the terms and conditions of the Creative Commons Attribution (CC BY) license (http://creativecommons.org/licenses/by/4.0/). 